\title{
Measurements of the charge ratio and polarization of cosmic-ray muons with the Super-Kamiokande detector
}

\author{
Hussain Kitagawa, ${ }^{a}{ }^{*}$ Yuuki Nakano ${ }^{b}$ and Yusuke Koshio ${ }^{a}$ on behalf of the \\ Super-Kamiokande Collaboration \\ (a complete list of authors can be found at the end of the proceedings) \\ ${ }^{a}$ Okayama University, Department of Physics, \\ Tsushimanaka, Kita-ku, Okayama, Japan \\ ${ }^{b}$ Kobe University, Department of Physics, \\ Rokko-dai, Nada-ku, Kobe, Japan \\ E-mail: kitagawa0913@s.okayama-u.ac.jp, ynakano@phys.sci.kobe-u.ac.jp, \\ koshio@okayama-u.ac.jp
}

Cosmic-ray muons arise from the showers of secondary particles produced via the interactions of primary cosmic particles with air nuclei at the top of the atmosphere. The interaction products, pions and kaons composing showers mostly decay to muons reflect the details of the hadronic interactions depending on their energy. Measurements of the charge ratio and polarization of cosmic-ray muons can be used to constrain high energy hadronic interaction models in the atmosphere. In this presentation, we will report the current status of the measurement of the charge ratio using data collected by the Super-Kamiokande (SK) detector located at a depth of $2700 \mathrm{~m}$ of water equivalent. Using the data taken in the fourth phase of SK (SK-IV), the charge ratio is measured to be $1.42 \pm 0.02$ (statistical uncertainty only), at the energy of $1.3 \mathrm{TeV}$. This result for the charge ratio is in good agreement with those previously obtained by deep underground experiments.

$37^{\text {th }}$ International Cosmic Ray Conference (ICRC 2021)

July 12th - 23rd, 2021

Online - Berlin, Germany

\footnotetext{
${ }^{*}$ Presenter
} 


\section{Introduction}

The framework of the neutrino oscillation has been established by various experimental measurements since 1980's. For the further precise measurements of the oscillation parameters, the uncertainties of atmospheric neutrinos flux should be reduced. To address this, the charge ratio of cosmic-ray is important to understand the energy dependence of the fraction of atmospheric neutrino flavors.

The cosmic-ray muons originate from decay of hadronic secondaries produced via interactions of primary cosmic-rays, such as proton and alpha particle. The muon charge ratio is defined as the ratio of the number of positive to negative charge atmospheric muons. Most of cosmic-ray primaries are positively charged, hence, more positive mesons such as pions and kaons will be produced in hadronic shower. In the previous measurements by the CMS [1] below $1 \mathrm{TeV} / c$ and OPERA [2] above $1 \mathrm{TeV} / c$, the charge ratio was approximately constant at $R\left(\mu^{+} / \mu^{-}\right) \approx 1.27$ up to $200 \mathrm{GeV} / c$ and then increases with higher momentum region. As energy increases, the fraction of muons come from decay of kaons increases because longer lifetime of pions more likely to interact with the atmosphere before decaying comparing to shorter lifetime kaons. The charge ratio strongly related with the energy distributions of atmospheric neutrinos because both of them are simultaneously produced. Therefore, it can be a useful tool to constrain the calculation of atmospheric neutrino flux.

In addition to this ratio, the polarization of cosmic-ray muons also related with the fraction of muons from kaon decays. The polarization directly reflects the kaons and pions production ratio of the secondary particles and this also provides input information for the simulation, which calculate the atmospheric neutrino fluxes.

\section{Super-Kamiokande detector}

Super-Kamiokande (SK) is the water Cherenkov detector located at $1000 \mathrm{~m}$ underground (2700 $\mathrm{m}$ water equivalent) in Kamioka mine in Japan [3]. The detector is consist of a cylindrical stainless steel tank, $39 \mathrm{~m}$ in diameter and $42 \mathrm{~m}$ in height. The tank contains a total of 50 kilotons of ultra-pure water. The inside of the tank is separated into an inner detector (ID) and an outer detector (OD). Between the ID and OD, there is a stainless steel support structure having a width of about $60 \mathrm{~cm}$ to accommodate the PMTs. It consists of 11,129 20-inch PMTs facing inward, and the space between the ID PMTs is covered with a black sheet to reduce unnecessary photon reflection. A fiducial volume of the ID is defined as the cylindrical volume $2 \mathrm{~m}$ inward from the ID wall and has a mass of 22.5 kilotons. The OD region has a width of about $2.2 \mathrm{~m}$ along the wall of the SK tank. It detects outgoing charged particles, and can also be used as a veto detector for cosmic-ray muons and low energy gamma rays from surrounding rocks. The fourth phase of the SK experiment (SK-IV) began in August 2008 with the new electronics and data acquisition systems [4]. The upgrade of electronic systems enlarge the time window of event trigger and contributed to the high detecting efficiency of the delayed signal of primary event. Experimental phases of SK are summarized in Table 1. 
Table 1: Experimental phases of SK. The livetimes are the duration of the observation after removing bad/calibration runs.

\begin{tabular}{ccccccc}
\hline \hline Phase & SK-I & SK-II & SK-III & SK-IV & SK-V & SK-VI \\
\hline Period (Start) & April 1996 & October 2002 & July 2006 & September 2008 & January 2019 & July 2020 \\
Period (End) & July 2001 & October 2005 & August 2008 & May 2018 & July 2020 & Running \\
Livetime [days] & 1496 & 791 & 548 & 2970 & 379 & - \\
ID PMTs & 11,146 & 5,182 & 11,129 & 11,129 & 11,129 & 11,129 \\
OD PMTs & 1,885 & 1,885 & 1,885 & 1,885 & 1,885 & 1,885 \\
PMT coverage [\%] & 40 & 19 & 40 & 40 & 40 & 40 \\
\hline \hline
\end{tabular}

\section{Data analysis}

The charge ratio of cosmic-ray muons at SK can be measured by counting the number of positive muons and negative muons from $\mu-e$ decay events. Cosmic-ray muons with energy of greater than $1.3 \mathrm{TeV}$ penetrate $1000 \mathrm{~m}$ of the length of rock and eventually reach to the SK tank at the rate of $2 \mathrm{~Hz}$. In the data analysis, the selection criteria for $\mu-e$ decay events were optimized by comparing the observed data with the MC simulation.

\subsection{Muon simulation}

For understand the detector's response to the muon decay process, we used the SK detector simulation based on GEANT3 tool-kit [5]. This simulation provides a common physical reaction for particle transformation in a water and electronic systems response. Thus, to evaluate the selection criteria for cosmic-ray muons observed in the detector, the whole muon decay process was performed by using this tool. In order to simulate muon decay events inside the tank, the generated energy range was adjusted from $0.1 \mathrm{MeV}$ to $20 \mathrm{GeV}$ to avoid muon events which penetrate the tank without decaying.

\subsection{Event reconstruction for cosmic-ray muons}

A pairs of stopped muon and decay electron ( $\mu-e$ decay events) are selected according to the following descriptions. For muon candidates, the total number of photoelectrons (p.e.) detected by ID PMTs is required to be greater than 1500 p.e. These muon candidates are reconstructed with a dedicated muon fitter [6], [7]. Muons are categorized in four classes, (I) Single through-going muons: events which penetrate the ID (II) Stopping muons: events which stopped inside the ID (III) Multiple muons: events with several tracks (IV) Corner clipping muons: events with a track length shorter than $7 \mathrm{~m}$ inside the ID. In this analysis, muon candidates regarded as stopping muons are used. In addition, to separate neutrino-induced muons from muon neutrino interactions in the rock around the detector, only down going muons with the zenith angle distribution respect to the detector height, $\theta<90^{\circ}$ are selected.

\subsection{Selection for decay electrons}

From the information of prompt muon event, decay electron candidates are selected by applying several reduction cuts. In this analysis, to distinguish the delayed signal from the primary event 


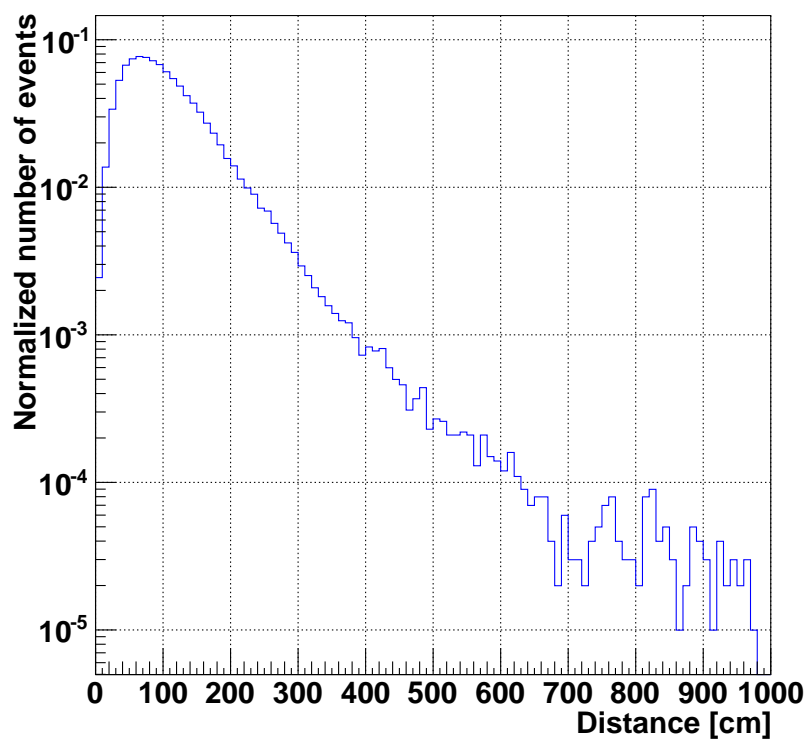

Figure 1: The reconstructed distance between the stop position of the muon and vertex position of the decay electron in the MC simulation.

which has broad time information, the time interval between the prompt event (muon) and the delayed event (electron) should satisfy $1.3 \mu \mathrm{sec}<t<30 \mu \mathrm{sec}$. The vertex position and the energy of decay electron candidates are reconstructed with an algorithm based on arrival time and the total number of hit PMTs. According to the reconstructed information of decay electrons, cuts criteria were determined for each parameter additionally. Fig. 1 shows the reconstructed distance between the stop position of the muon and vertex position of the decay electron in the MC simulation. It can be seen from Fig. 1 that most events are reconstructed in the shorter region less than $300 \mathrm{~cm}^{1}$. To remove low energy backgrounds from nuclear capture, energy threshold for decay electron is set to be greater than $8 \mathrm{MeV}$. The distribution of the reconstructed energy and the time information of selected decay electron events obtained from the MC simulation are shown in Fig. 2.

\section{Results}

Due to much small residual magnetic field in the water tank [8], the SK can not distinguish the charge of the cosmic-ray muons in principle. However, some of negative muons are captured by ${ }^{16} \mathrm{O}$ nuclei in the detector, this results in the shorter lifetime of negative muon than that in vacuum. The lifetime difference between the positive and the negative muons allows to estimate the ratio of the muon by measuring the time of the muon decay.

The decay curve of stopping muons obtained from the time interval between parent muon event and delayed electron events are shown in Fig. 3. Positive muons are free from nuclear capture and its lifetime muon is equal to $\tau_{\mu}$ in vacuum, which corresponds to $\tau_{\mu}=2.1969811 \pm 0.0000022 \mu \mathrm{sec}$ [9].

\footnotetext{
${ }^{1}$ The vertex position resolution for a $15 \mathrm{MeV}$ electron is $42 \mathrm{~cm}$. The low energy electron pass through a few $\mathrm{cm}$ in ID, hence, the track length is negligible.
} 


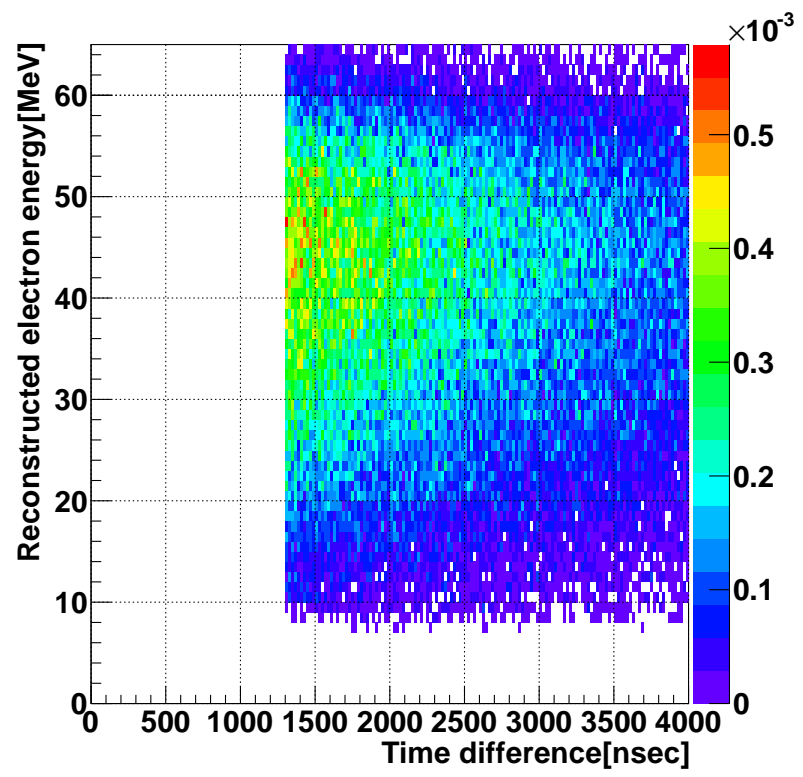

Figure 2: The reconstructed energy and time distribution of decay electrons in the MC simulation. The color plots represent the number of events normalized with total events after reduction cuts.

Conversely, a negative muon forms muonic atom in a medium and its lifetime become shorter than $\tau_{\mu^{+}}$because of muon capture by nucleus. Measurements of the lifetime in various matters for negative muons have been performed in the previous measurements [10]. The mean lifetime of negative muons in pure water is found to be $\tau_{\mu^{-}}=1.7954 \pm 0.0020 \mu \mathrm{sec}$ [10]. Muons measured in the detector, consist of two components, $\tau_{\mu^{+}}$and $\tau_{\mu^{-}}$, therefore, the lifetime spectrum is fitted by the sum of two exponential functions,

$$
N(t-(t+\Delta t))=N_{+}\left\{1-\exp \left(-\frac{\Delta t}{\tau_{\mu^{+}}}\right)\right\} \exp \left(-\frac{t}{\tau_{\mu^{+}}}\right)+N_{-}\left\{1-\exp \left(-\frac{\Delta t}{\tau_{\mu^{-}}}\right)\right\} \exp \left(-\frac{t}{\tau_{\mu^{-}}}\right),
$$

where $\Delta t$ is the binning width of the decay curve histogram set to be $0.1 \mu$ sec in this study, and $N_{+}, N_{-}$are the total number of detected positive muons and negative muons, respectively. Since the water can be assumed as ${ }^{16} \mathrm{O}$, the nuclear capture fraction for negative muons in water is $\Lambda_{c}=0.184 \pm 0.001$ [10]. Considering nuclear capture effects on negative muons, the charge ratio $R\left(\mu^{+} / \mu^{-}\right)$can be expressed by,

$$
R\left(\mu^{+} / \mu^{-}\right)=\frac{N_{+}}{N_{-} /\left(1-\Lambda_{c}\right)} .
$$

From the fitting of the decay curve of $\mu-e$ decay events observed in 280 days of effective live time in a 2017's data, $N_{+}$and $N_{-}$were obtained as Table 2. Hence, the charge ratio is $1.38 \pm 0.05$ (statistical uncertainty only) from 2017's data. The yearly variation of the charge ratio in the data period of SK-IV was calculated in similar method likewise. Consequently, the charge ratio of cosmic-ray muons at the energy of $1.3 \mathrm{TeV}$ is found to be, $1.42 \pm 0.02$ (statistical uncertainty only). The combined data points are plotted in Fig. 4 together with the previous measurement at 
Table 2: The results of $N_{+}, N_{-}$and the charge ratio $R\left(\mu^{+} / \mu^{-}\right)$observed in data (2017). In this study, only statistical uncertainty is considered.

\begin{tabular}{c|c}
\hline \hline & Example in 2017 (280.9 days) \\
\hline$N_{+}$ & $360869.4 \pm 6155.1$ \\
$N_{-}$ & $213995.8 \pm 7372.6$ \\
$R\left(\mu^{+} / \mu^{-}\right)$ & $1.38 \pm 0.05$ \\
\hline \hline
\end{tabular}

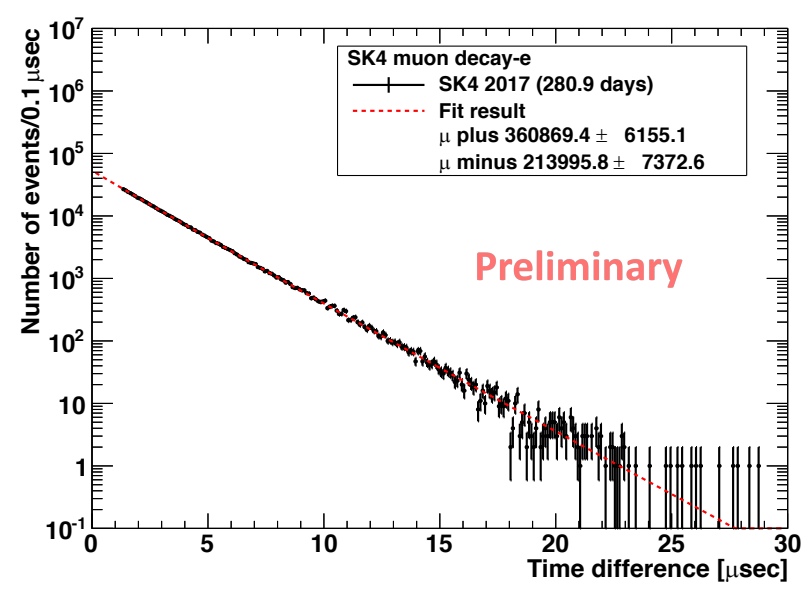

Figure 3: The example of decay curve of stopping muons in the SK detector in 2017 data. The red dashed line is obtained by fitting a two component decay function with lifetimes of $2.1969811 \pm 0.0000022 \mu \mathrm{sec}$ [9] for $\mu^{+}$and $1.7954 \pm 0.0020 \mu \sec [10]$ for $\mu^{-}$in pure water.

Kamiokande-II [11]. The total charge ratio in SK-IV phase is slightly higher comparing to that of Kamiokande-II. However, the SK-IV result is consistent with the Kamiokande-II's result within their uncertainties.

\section{Summary and Future prospects}

We have measured the charge ratio of positive to negative cosmic-ray muons at the energy of $1.3 \mathrm{TeV}$, through the analysis of the decay curve of stopping muons in the SK detector using data collected from 2008 to 2018. In conclusion, the charge ratio is found to be $1.42 \pm 0.02$ (statistical uncertainty only), and the result is in agreement with past measurements at Kamiokande-II within their uncertainties. As the future prospects, we will measure the charge ratio by combining the whole data set of SK-I, II, and III with high statistics, including systematic uncertainties. This result will allow us to search for the correlation between the time variation of the charge ratio and the solar activity. In addition, analysis for spin polarization of cosmic-ray muons is also on-going. 


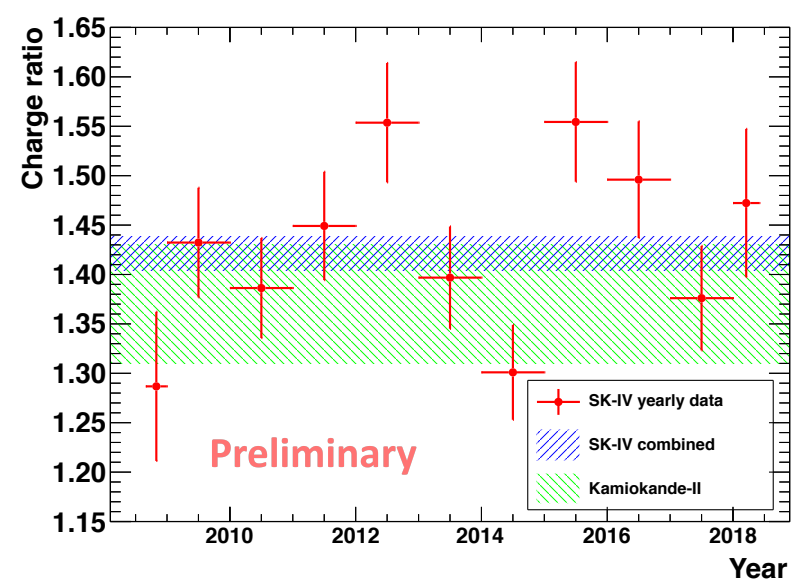

Figure 4: Yearly time variation of the charge ratio of $1.3 \mathrm{TeV}$ cosmic-ray muons from 2008 to 2018 together with the result from the Kamiokande-II. The blue band area shows the average of muon charge ratio in SK-IV period (from 2008 October to 2018 May) and green band is the result from Kamiokande-II [11].

\section{References}

[1] V. Khachatryan, et al.: Phys. Lett. B 692, 83 (2010).

[2] N. Agafonova, et al.: Eur. Phys. J. C 67, 25 (2010).

[3] S. Fukuda, et al.: Nucl. Instrum. Meth. A 501, 418 (2003).

[4] S. Yamada, et al:: IEEE Transactions on Nuclear Science 57, 2 (2010).

[5] R. Brun, et al.: GEANT: Detector Description and Simulation Tool, CERN Program LibraryW5013 (1994).

[6] S. Desai, Ph.D. thesis, Boston University, (2004).

[7] Z. Conner, Ph.D. thesis, University of Maryland, (1997).

[8] K. Abe, et al.: Nucl. Instrum. Meth. A 737, 253 (2014).

[9] P. A. Zyla, et al.: (Particle Data Group), Prog.Theor.Exp.Phys. 2020, 083 C01 (2020).

[10] T. Suzuki, D.F. Measday, and J.P. Roalsvig, Phys. Rev. C 35, 2212 (1987).

[11] M. Yamada, et al.: Phys. Rev. D 44, 617 (1991). 


\section{Full Authors List: Super-Kamiokande Collaboration}

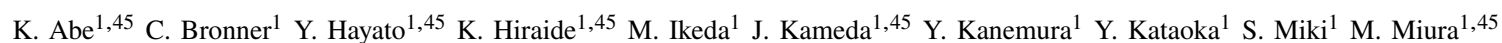
S. Moriyama ${ }^{1,45}$ Y. Nagao ${ }^{1}$ M. Nakahata ${ }^{1,45}$ S. Nakayama ${ }^{1,45}$ K. Okamoto $^{1}$ G. Pronost ${ }^{1}$ H. Sekiya ${ }^{1,45}$ M. Shiozawa ${ }^{1,45}$ Y. Sonoda $^{1}$ Y. Suzuki ${ }^{1}$ A. Takeda ${ }^{1,45}$ Y. Takemoto ${ }^{1}$ A. Takenaka ${ }^{1}$ H. Tanaka ${ }^{1}$ S. Watanabe ${ }^{1}$ T. Yano ${ }^{1}$ S. Han ${ }^{2}$ T. Kajita ${ }^{2,45}$ K. Okumura ${ }^{2,45}$ T. Tashiro ${ }^{2}$ J. Xia ${ }^{2}$ G. D. Megias ${ }^{3}$ L. Labarga ${ }^{4}$ Ll. Marti ${ }^{4}$ B. Zaldivar ${ }^{4}$ B. W. Pointon ${ }^{6,49}$ E. Kearns ${ }^{5,45}$ J. L. Raaf ${ }^{5}$ L. Wan ${ }^{5}$ T. Wester ${ }^{5}$

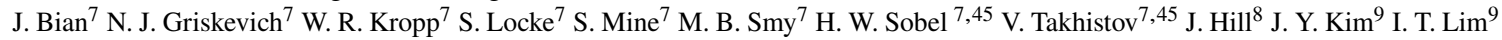
R. G. Park ${ }^{9}$ B. Bodur ${ }^{10}$ K. Scholberg ${ }^{10}$ C. W. Walter ${ }^{10,45}$ L. Bernard ${ }^{11}$ A. Coffani ${ }^{11}$ O. Drapier ${ }^{11}$ S. El Hedri ${ }^{11}$ A. Giampaolo ${ }^{11}$ M. Gonin ${ }^{11}$ Th. A. Mueller ${ }^{11}$ P. Paganini ${ }^{11}$ B. Quilain ${ }^{11}$ T. Ishizuka ${ }^{12}$ T. Nakamura ${ }^{13}$ J. S. Jang ${ }^{14}$ J. G. Learned ${ }^{15}$ S. Cao ${ }^{16}$ L. H. V. Anthony ${ }^{17}$ D. Martin ${ }^{17}$ M. Scott ${ }^{17}$ A. A. Sztuc $^{17}$ Y. Uchida ${ }^{17}$ V. Berardi ${ }^{18}$ M. G. Catanesi ${ }^{18}$ E. Radicioni ${ }^{18}$ N. F. Calabria ${ }^{19}$ L. N. Machado ${ }^{19}$ G. De Rosa $^{19}$ G. Collazuol ${ }^{20}$ F. Iacob $^{20}$ M. Lamoureux ${ }^{20}$ M. Mattiazzi ${ }^{20}$ N. Ospina ${ }^{20}$ L. Ludovici ${ }^{21}$ Y. Maekawa $^{22}$ Y. Nishimura ${ }^{22}$ M. Friend ${ }^{23}$ T. Hasegawa ${ }^{23}$ T. Ishida ${ }^{23}$ T. Kobayashi ${ }^{23}$ M. Jakkapu ${ }^{23}$ T. Matsubara ${ }^{23}$ T. Nakadaira ${ }^{23}$ K. Nakamura ${ }^{23,45}$ Y. Oyama ${ }^{23}$ K. Sakashita ${ }^{23}$ T. Sekiguchi ${ }^{23}$ T. Tsukamoto ${ }^{23}$ T. Boschi ${ }^{24}$ F. Di Lodovico ${ }^{24}$ J. Gao $^{24}$ J. Migenda $^{24}$ M. Taani ${ }^{24}$ S. Zsoldos $^{24}$

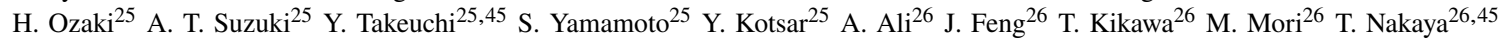
R. A. Wendell ${ }^{26,45}$ K. Yasutome ${ }^{26}$ P. Fernandez ${ }^{28}$ N. McCauley ${ }^{28}$ P. Mehta $^{28}$ K. M. Tsui ${ }^{27}$ Y. Fukuda $^{28}$ Y. Itow $^{29,30}$ H. Menjo $^{29}$ K. Sato $^{29}$ J. Lagoda ${ }^{31}$ S. M. Lakshmi ${ }^{31}$ P. Mijakowski ${ }^{31}$ J. Zalipska ${ }^{31}$ J. Jiang $^{32}$ C. K. Jung ${ }^{32}$ C. Vilela ${ }^{32}$ M. J. Wilking ${ }^{32}$ C. Yanagisawa ${ }^{32}$ M. Harada ${ }^{33}$ H. Ishino ${ }^{33}$ S. Ito ${ }^{33}$ W. Ma ${ }^{33}$ N. Piplani ${ }^{33}$ S. Sakai ${ }^{33}$ G. Barr ${ }^{34}$ D. Barrow ${ }^{34}$ L. Cook ${ }^{34,45}$ A. Goldsack ${ }^{34,45}$ S. Samani ${ }^{34}$ D. Wark ${ }^{34,39}$ F. Nova ${ }^{35}$ J. Y. Yang ${ }^{36}$ S. J. Jenkins ${ }^{37}$ M. Malek ${ }^{37}$ J. M. McElwee ${ }^{37}$ O. Stone ${ }^{37}$ M. D. Thiesse ${ }^{37}$ L. F. Thompson ${ }^{37}$ H. Okazawa ${ }^{38}$ S. B. Kim $^{40}$ I. Yu ${ }^{40}$ A. K. Ichikawa ${ }^{41}$ K. Nakamura ${ }^{41}$ K. Nishijima ${ }^{42}$ M. Koshiba ${ }^{43}$ K. Iwamoto ${ }^{44}$ Y. Nakajima ${ }^{44}$ N. Ogawa ${ }^{44}$ M. Yokoyama ${ }^{44,45}$ K. Martens ${ }^{45}$ M. R. Vagins ${ }^{45,7}$ M. Kuze ${ }^{46}$ S. Izumiyama ${ }^{46}$ M. Inomoto ${ }^{47}$ M. Ishitsuka ${ }^{47}$ H. Ito $^{47}$ T. Kinoshita ${ }^{47}$ R. Matsumoto ${ }^{47}$ M. Shinoki ${ }^{47}$ T. Suganuma ${ }^{47}$ J. F. Martin ${ }^{48}$ H. A. Tanaka ${ }^{48}$ T. Towstego ${ }^{48}$ R. Akutsu ${ }^{49}$ P. de Perio ${ }^{49}$ M. Hartz ${ }^{49}$ A. Konaka ${ }^{49}$ N. W. Prouse ${ }^{49}$ S. Chen $^{50}$ B. D. $\mathrm{Xu}^{50}$ M. Posiadala-Zezula ${ }^{51}$ D. Hadley ${ }^{52}$ M. O'Flaherty ${ }^{52}$ B. Richards ${ }^{52}$ B. Jamieson ${ }^{53}$ J. Walker ${ }^{53}$ A. Minamino ${ }^{54}$ G. Pintaudi ${ }^{54}$ S. Sano ${ }^{54}$ R. Sasaki ${ }^{54}$

Kamioka Observatory, Institute for Cosmic Ray Research, University of Tokyo, Kamioka, Gifu 506-1205, Japan.

${ }^{2}$ Research Center for Cosmic Neutrinos, Institute for Cosmic Ray Research, University of Tokyo, Kashiwa, Chiba 277-8582, Japan.

${ }^{3}$ Institute for Cosmic Ray Research, University of Tokyo, Kashiwa, Chiba 277-8582, Japan.

${ }^{4}$ Department of Theoretical Physics, University Autonoma Madrid, 28049 Madrid, Spain.

${ }^{5}$ Department of Physics, Boston University, Boston, MA 02215, USA.

${ }^{6}$ Department of Physics, British Columbia Institute of Technology, Burnaby, BC, V5G 3H2, Canada.

${ }^{7}$ Department of Physics and Astronomy, University of California, Irvine, Irvine, CA 92697-4575, USA

${ }^{8}$ Department of Physics, California State University, Dominguez Hills, Carson, CA 90747, USA.

${ }^{9}$ Institute for Universe and Elementary Particles, Chonnam National University, Gwangju 61186, Korea

${ }^{10}$ Department of Physics, Duke University, Durham NC 27708, USA.

${ }^{11}$ Ecole Polytechnique, IN2P3-CNRS, Laboratoire Leprince-Ringuet, F-91120 Palaiseau, France.

${ }_{12}^{12}$ Junior College, Fukuoka Institute of Technology, Fukuoka, Fukuoka 811-0295, Japan.

${ }^{13}$ Department of Physics, Gifu University, Gifu, Gifu 501-1193, Japan.

${ }^{14}$ GIST College, Gwangju Institute of Science and Technology, Gwangju 500-712, Korea.

${ }^{15}$ Department of Physics and Astronomy, University of Hawaii, Honolulu, HI 96822, USA

${ }^{16}$ Institute For Interdisciplinary Research in Science and Education, ICISE, Quy Nhon, 55121, Vietnam.

${ }^{17}$ Department of Physics, Imperial College London, London, SW7 2AZ, United Kingdom

${ }^{18}$ Dipartimento Interuniversitario di Fisica, INFN Sezione di Bari and Università e Politecnico di Bari, I-70125, Bari, Italy.

${ }^{19}$ Dipartimento di Fisica, INFN Sezione di Napoli and Università di Napoli, I-80126, Napoli, Italy

${ }^{20}$ Dipartimento di Fisica, INFN Sezione di Padova and Università di Padova, I-35131, Padova, Italy.

${ }^{21}$ INFN Sezione di Roma and Università di Roma "La Sapienza", I-00185, Roma, Italy.

${ }^{22}$ Department of Physics, Keio University, Yokohama, Kanagawa, 223-8522, Japan.

${ }^{23}$ High Energy Accelerator Research Organization (KEK), Tsukuba, Ibaraki 305-0801, Japan

${ }^{24}$ Department of Physics, King's College London, London, WC2R 2LS, UK

${ }^{25}$ Department of Physics, Kobe University, Kobe, Hyogo 657-8501, Japan.

${ }^{26}$ Department of Physics, Kyoto University, Kyoto, Kyoto 606-8502, Japa

${ }^{27}$ Department of Physics, University of Liverpool, Liverpool, L69 7ZE, United Kingdom.

${ }^{28}$ Department of Physics, Miyagi University of Education, Sendai, Miyagi 980-0845, Japan.

${ }^{29}$ Institute for Space-Earth Environmental Research, Nagoya University, Nagoya, Aichi 464-8602, Japan.

${ }^{30}$ Kobayashi-Maskawa Institute for the Origin of Particles and the Universe, Nagoya University, Nagoya, Aichi 464-8602, Japan.

${ }_{31}^{31}$ National Centre For Nuclear Research, 02-093 Warsaw, Poland.

${ }^{32}$ Department of Physics and Astronomy, State University of New York at Stony Brook, NY 11794-3800, USA

${ }^{33}$ Department of Physics, Okayama University, Okayama, Okayama 700-8530, Japan

${ }^{34}$ Department of Physics, Oxford University, Oxford, OX1 3PU, United Kingdom.

${ }^{35}$ Rutherford Appleton Laboratory, Harwell, Oxford, OX11 0QX, UK.

${ }^{36}$ Department of Physics, Seoul National University, Seoul 151-742, Korea.

${ }^{37}$ Department of Physics and Astronomy, University of Sheffield, S3 7RH, Sheffield, United Kingdom.

${ }^{38}$ Department of Informatics in Social Welfare, Shizuoka University of Welfare, Yaizu, Shizuoka, 425-8611, Japan

${ }^{39}$ STFC, Rutherford Appleton Laboratory, Harwell Oxford, and Daresbury Laboratory, Warrington, OX11 0QX, United Kingdom.

${ }^{40}$ Department of Physics, Sungkyunkwan University, Suwon 440-746, Korea.

${ }^{41}$ Department of Physics, Faculty of Science, Tohoku University, Sendai, Miyagi, 980-8578, Japan.

${ }^{42}$ Department of Physics, Tokai University, Hiratsuka, Kanagawa 259-1292, Japan.

${ }^{43}$ The University of Tokyo, Bunkyo, Tokyo 113-0033, Japan.

${ }^{44}$ Department of Physics, University of Tokyo, Bunkyo, Tokyo 113-0033, Japan

${ }^{45}$ Kavli Institute for the Physics and Mathematics of the Universe (WPI), The University of Tokyo Institutes for Advanced Study, University of Tokyo, Kashiwa, Chiba 277-8583, Japan. ${ }^{46}$ Department of Physics,Tokyo Institute of Technology, Meguro, Tokyo 152-8551, Japan.

${ }^{47}$ Department of Physics, Faculty of Science and Technology, Tokyo University of Science, Noda, Chiba 278-8510, Japan

${ }^{48}$ Department of Physics, University of Toronto, ON, M5S 1A7, Canada.

${ }^{49}$ TRIUMF, 4004 Wesbrook Mall, Vancouver, BC, V6T2A3, Canada.

${ }^{50}$ Department of Engineering Physics, Tsinghua University, Beijing, 100084, China.

${ }^{51}$ Faculty of Physics, University of Warsaw, Warsaw, 02-093, Poland.

${ }^{52}$ Department of Physics, University of Warwick, Coventry, CV4 7AL, UK.

${ }^{53}$ Department of Physics, University of Winnipeg, MB R3J 3L8, Canada.

${ }^{54}$ Department of Physics, Yokohama National University, Yokohama, Kanagawa, 240-8501, Japan.

${ }^{2}$ also at BMCC/CUNY, Science Department, New York, New York, 1007, USA.

${ }^{3}$ Deceased. 